\title{
Various Bronchiolar Lesions Accompanied by Idiopathic Pleuroparenchymal Fibroelastosis with a Usual Interstitial Pneumonia Pattern Demonstrating Acute Exacerbation
}

\author{
Atsushi Miyamoto ${ }^{1}$, Hironori Uruga ${ }^{1,2}$, Nasa Morokawa ${ }^{1}$, Shuhei Moriguchi ${ }^{1}$, Yui Takahashi ${ }^{1}$, \\ Kazumasa Ogawa ${ }^{1}$, Kyoko Murase ${ }^{1}$, Shigeo Hanada ${ }^{1,2}$, Hisashi Takaya ${ }^{1}$, Atsuko Kurosaki ${ }^{3}$, \\ Takeshi Fujii ${ }^{2,4}$, Eugene J. Mark ${ }^{5}$ and Kazuma Kishi ${ }^{1,2}$
}

\begin{abstract}
:
A 63-year-old man presented with persistent cough and progressive dyspnea. Computed tomography showed irregular pleural thickening and fibrotic changes with volume loss in the upper lobes, and subtle reticulation in the lower lobes. Pleuroparenchymal fibroelastosis (PPFE) was diagnosed based on the findings of a surgical lung biopsy. Bronchiolar lesions, including proliferative bronchiolitis, constrictive bronchiolitis obliterans, and peribronchiolar metaplasia were evident on pathology. A usual interstitial pneumonia (UIP) pattern was also observed in the lower lobes. Three weeks after the biopsy, an acute exacerbation occurred. We herein describe a rare case of idiopathic PPFE with various bronchiolar lesions and a UIP pattern in which an acute exacerbation developed.
\end{abstract}

Key words: acute exacerbation, constrictive bronchiolitis obliterans, idiopathic interstitial pneumonia, idiopathic pleuroparenchymal fibroelastosis, usual interstitial pneumonia

(Intern Med 58: 1321-1328, 2019)

(DOI: 10.2169/internalmedicine.1649-18)

\section{Introduction}

Idiopathic pleuroparenchymal fibroelastosis (PPFE) is a rare emerging form of idiopathic interstitial pneumonia that is pathologically characterized by alveolar collapse with parenchymal fibrosis and elastosis in the subpleural area, predominantly occurring in the upper lobes (1-3). It was initially reported in the Japanese literature by Amitani et al. (4), as idiopathic pulmonary upper lobe fibrosis.

A PPFE pattern in the upper lobes concomitant with a usual interstitial pneumonia (UIP) pattern in the lower lobes has been described as a feature that distinguishes idiopathic PPFE from pure idiopathic pulmonary fibrosis (IPF) $(1,5)$. The clinical course of idiopathic PPFE is gradually progressive; however, there is only one case report of an acute ex- acerbation without any interstitial fibrotic lesion in the lower lobes (6). A few cases of acute exacerbations of PPFE due to pre-existing fibrosis with a UIP pattern or an unclassifiable pattern in the lower lobes have been reported (7). However, there have been no detailed case reports describing the clinical course of such cases, including the physical and radiologic features and treatment of such cases.

Little is known about the associations between a pathologically identified PPFE pattern and various bronchiolar lesions, such as proliferative bronchiolitis, constrictive bronchiolitis obliterans, and bronchiolar metaplasia in patients with idiopathic PPFE. The coexistence of PPFE and bronchiolar lesions has only been clearly described in the context of allografted lung in transplant recipients and associated chronic graft rejection (8).

We herein describe a rare case of a patient with pa-

\footnotetext{
${ }^{1}$ Department of Respiratory Medicine, Respiratory Center, Toranomon Hospital, Japan, ${ }^{2}$ Okinaka Memorial Institute for Medical Research, Japan, ${ }^{3}$ Department of Diagnostic Radiology, Fukujuji Hospital, Japan Anti-tuberculosis Association, Japan, ${ }^{4}$ Department of Pathology, Toranomon Hospital, Japan and ${ }^{5}$ Department of Pathology, Massachusetts General Hospital, USA

Received: June 5, 2018; Accepted: October 8, 2018; Advance Publication by J-STAGE: December 18, 2018

Correspondence to Dr. Atsushi Miyamoto, atsushimotty@gmail.com
} 
Table 1. Clinical Data on Initial Admission before Biopsy.

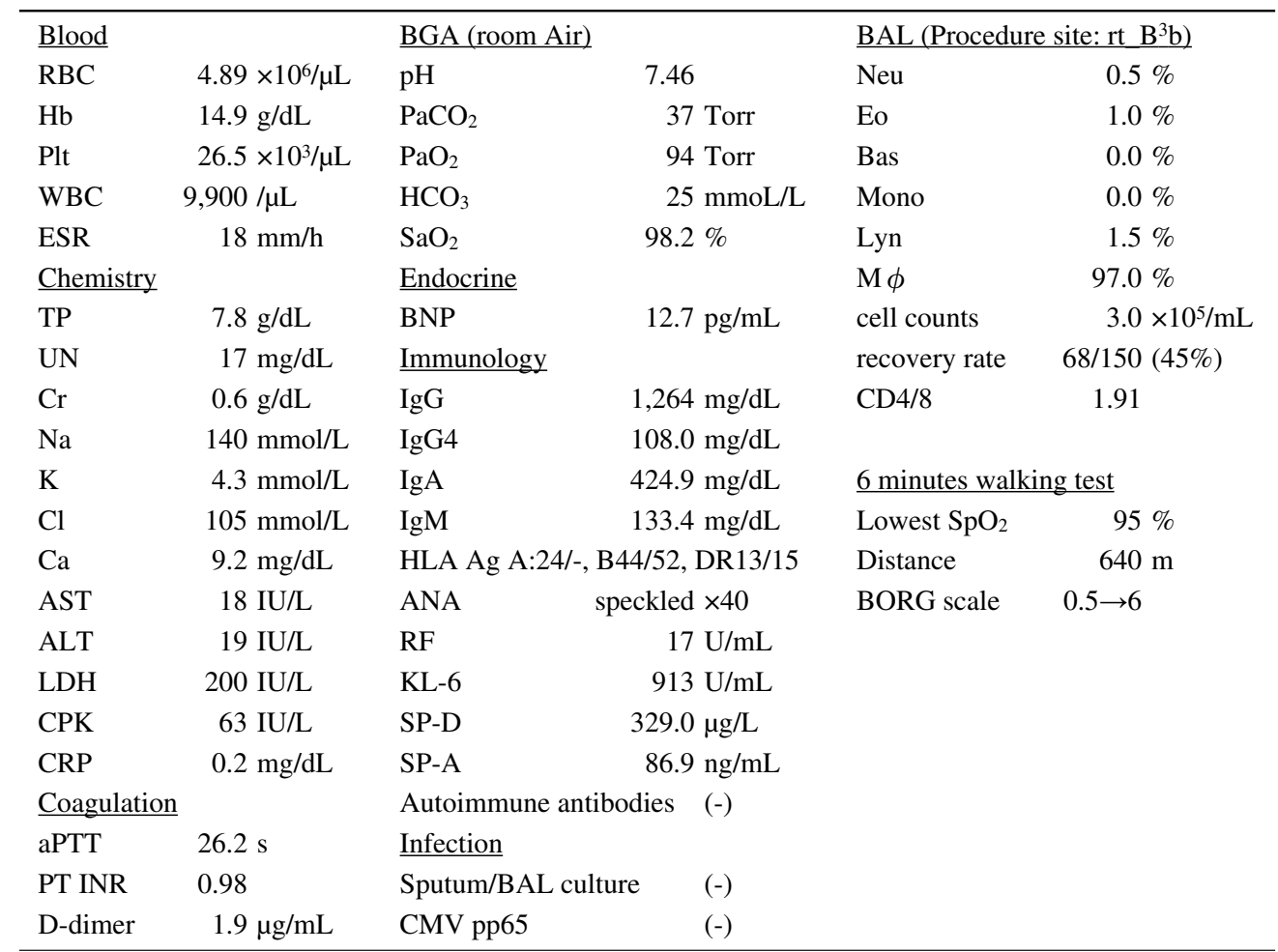

Ag: antigen, ALT: alanine aminotransferase, ANA: anti-nuclear antigen, aPTT: activated partial thromboplastin time, AST: asparate aminotransferase, BAL: bronchoalveolar lavage, Bas: basophil, BC: blood count, BGA: blood gas analysis, BNP: B-type natriuretic peptide, Ca: calcium, CD: cluster designation, Cl: chloride, CMV: cytomegarovirus, CPK: creatine phosphokinase, Cr: creatinine, CRP: C-reactive protein, Eo: eosinophil, ESR: erythrocyte segmentation rate, $\mathrm{Hb}$ : hemoglobin, $\mathrm{HCO}_{3}$ : bicarbonate, $\mathrm{HLA}$ : human leukocyte antigen, $\mathrm{IgA}$ : immunoglobulin A, IgG: immunoglobulin G, IgM: immunoglobulin M, K: potassium, KL-6: Krebs von den Lungen 6, LDH: lactate dehydrogenase, Lyn: lymphocyte, $\mathrm{M} \phi$ : macrophage, Na: sodium, Neu: neutrophil, $\mathrm{PaCO}_{2}$ : partial pressure of carbon dioxide in arterial blood, $\mathrm{PaO}_{2}$ : partial pressure of oxygen in arterial blood, Plt: platelet count, PT-INR: prothrombin time-International Normalized Ratio, RBC: red blood cell, RF: rheumatoid factor, SP-A: surfactant protein A, SP-D: surfactant protein D, $\mathrm{SpO}_{2}$ : arterial oxygen saturation of pulse oxymetry, TP: total protein, UN: urea nitrogen, WBC: white blood cell

thologically confirmed PPFE, a UIP pattern, and various accompanying bronchiolar lesions, who developed an acute exacerbation after surgical lung biopsy.

\section{Case Report}

\section{Clinical course}

A 63-year-old man presented to our department with a persistent cough and progressive dyspnea that had developed over the previous 6 months. Seven years before his initial admission to our hospital, chest X-ray abnormalities with an upper lung field predominance had been identified. No definitive diagnosis had been made at that time. The patient was an electrician and a former light smoker (8 pack-year). He reported no memory of inhaling any unusual dust or chemicals. His body mass index was $21.6 \mathrm{~kg} / \mathrm{m}^{2}$ (body weight, $58.0 \mathrm{~kg}$; height, $164 \mathrm{~cm}$ ); no family history of interstitial fibrosis was noted and the patient had not previously received lung or bone marrow transplantation, chemotherapy, or radiation therapy. The patient's clinical data, including the results of laboratory tests, a 6-minute walking test, and bronchial alveolar lavage are shown in Table 1. The partial pressure of oxygen in the arterial blood $\left(\mathrm{PaO}_{2}\right)$ was 94 Torr (room air). Although a serum analysis revealed antinuclear antibody positivity (low titer), and slight elevation of rheumatoid factor with high gamma immunoglobulinemia, no definite diagnosis of a collagen vascular disease such as ankylosing spondylitis or rheumatoid arthritis could be made. A chest X-ray revealed a reduced lung volume with an upper lung field predominance and bilateral reticular opacities and focal infiltrates that expanded from the upper to lower lung fields; this was demonstrated by comparison with a chest X-ray image obtained 6 months before admission (Fig. 1). Chest computed tomography (CT) suggested irregular pleural thickening with fibrotic change (Fig. 2a-c). In addition, a mixture of reticular opacity with focal consolidation was seen at the base of the lung (Fig. 2d). The ratio of the anteroposterior diameter of the thoracic cage to the transverse diameter of the thoracic cage - which was measured to investigate the flattened thoracic cage - was 0.568 (9). Pulmonary function testing (PFT) clearly revealed a restrictive ventilatory defect with impaired gas exchange (Fig. 3). The predicted $\dot{V}_{25}\left(\%_{25}\right)$ was relatively low in com- 

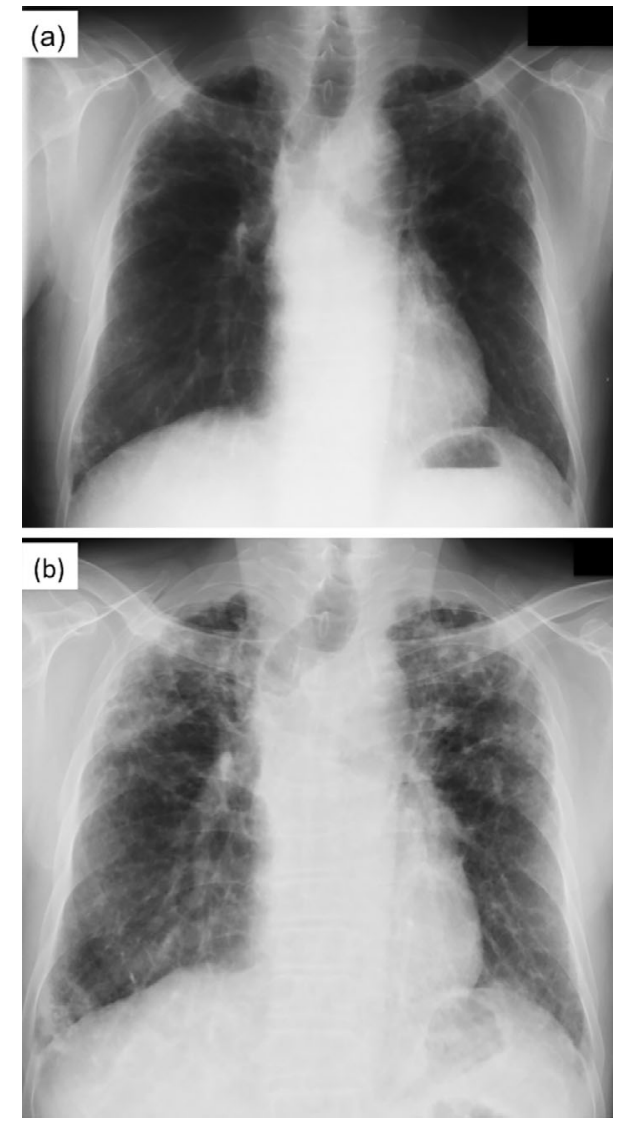

Figure 1. Chest X-ray images obtained six months before admission (a) and on admission (b). Pre-existing upper lung predominant reticulo-nodular opacities and the reduced volume of the upper lung field were seen. According to the worsening of persistent cough and dyspnea, the interstitial abnormalities deteriorated, majorly expanding to the lower lung fields.

parison to the predicted $\dot{\mathrm{V}}_{50}\left(\% \dot{\mathrm{V}}_{50}\right)$, which led to the concave flow-volume loop during the expiration phase of $25 \%$ to $75 \%$ of forced vital capacity (FVC). The forced expiratory volume in one second $\left(\mathrm{FEV}_{1}\right) / \mathrm{FVC}$ was normal but the predicted $\mathrm{FEV}_{1}\left(\% \mathrm{FEV}_{1}\right)$ was mildly declined. Lung biopsy using video-assisted thoracoscopic surgery was performed from the left $\mathrm{S}^{1+2}$ and $\mathrm{S}^{9}$.

At three weeks after surgical biopsy, the patient was admitted to our hospital due to rapidly worsening dyspnea on exertion. Chest CT revealed newly developed ground-glass opacities extending throughout the lungs with a right side predominance (Fig. 2e-h). The patient's $\mathrm{PaO}_{2}$ value declined to 75 Torr (room air). The serum levels of Krebs von den Lungen-6 and surfactant protein-D were higher than those just before biopsy $(1,290 \mathrm{U} / \mathrm{mL}$ and $459.0 \mu \mathrm{g} / \mathrm{L}$, respectively). Bacteriological cultures of expectorated sputum and blood yielded negative results. No physical findings suggestive of heart failure or infection, such as pitting edema in the lower extremities, or purulent sputum were observed. The serum levels of D-dimer and B-type natriuretic peptide were not suggestive of pulmonary thromboembolism (1.6 $\mu \mathrm{g} / \mathrm{mL})$ and heart failure $(6.6 \mathrm{pg} / \mathrm{mL})$, respectively. Thus, no alternative causes for the development of acute deterioration of his respiratory status, such as acute pulmonary edema, pulmonary infection, or pulmonary embolism were identified. Accordingly, we determined that an acute exacerbation had occurred $(10,11)$. Steroid pulse therapy [methylprednisolone $(1,000 \mathrm{mg} /$ day for 3 consecutive days)], followed by oral prednisolone $(20 \mathrm{mg} /$ day $)$ and cyclosporine A $(100 \mathrm{mg} /$ day) were initiated, after which his respiratory status and radiographic parameters gradually recovered. As an empiric antibiotic therapy, piperacillin/tazobactam was intravenously administered for the initial 14 days.

\section{The histopathological findings}

A panoramic view from the lower lobe in $S^{9}$ with hematoxylin and eosin staining revealed a UIP pattern, including spatial heterogeneity with subpleural/paraseptal (perilobular) fibrosis, and dense fibrosis accompanying architectural remodeling (Fig. 4a). There was an abrupt change from abnormal scarred lung to normal lung (i.e., patchwork pattern), and fibrosis with perilobular distribution and numerous fibroblastic foci, which was consistent with UIP (Fig. 4a, 5a-c). In an upper lobe, marked fibroelastosis with alveolar collapse in the subpleural airspace was present with a band-like distribution (Fig. 4b, 5d and e). In both upper and lower lobes, various membranous bronchiolar lesions were identified within the unaffected lung parenchyma, and they were clearly spatially separated from the fibroelastosis due to a PPFE pattern or dense fibrosis due to a UIP pattern (Fig. 5f and 1). The bronchiolar lesions included: 1) constrictive bronchiolitis accompanied by obstructive pneumonia with exudate and foamy macrophage infiltration into the alveolar spaces in the peripheral lobule (Fig. 5f-i); 2) proliferative bronchiolitis, that is, intraductal polypoid fibrosis mainly distributed in the bronchiolar lumen with quite limited extension to the adjacent peribronchiolar alveolar airspaces (Fig. 5j and k) (12); and 3) peribronchiolar metaplasia (Fig. 5l). No granulomas suggestive of any inhaled particles or apparent pigmented macrophages were identified.

\section{Discussion}

The current case was clinically characterized by the development of an acute exacerbation after surgical lung biopsy. Acute exacerbations typically occurs in $\operatorname{IPF}(10,11)$. Less commonly, chronic fibrosing interstitial lung diseases other than IPF are associated with the development of acute exacerbations (13). Nei et al. (6) described a case of idiopathic PPFE without any interstitial fibrotic lesions in the lower lobes, in which an acute exacerbation occurred. A few recently published studies have reported that $13.5-18.0 \%$ of PPFE patients experienced an acute exacerbation of their condition $(7,14,15)$. Enomoto et al. (7) reported that in two PPFE patients with acute exacerbations, the interstitial fibrosis in the lower lobes showed a UIP pattern and an unclassifiable pattern, respectively. Oda et al. (5) reported that one of nine $(11.1 \%)$ patients with PPFE and a UIP pattern experienced an acute exacerbation. A UIP pattern has been re- 



Figure 2. Chest computed tomography. (a-d) Just before the surgical lung biopsy. (e-g) Just after the diagnosis of the acute exacerbation.

ported to be a risk factor for an acute exacerbation after thoracic surgery (16). Thus, regardless of the presence or absence of fibrotic lesions in the lower lobes, physicians should be aware that acute exacerbations can occur in patients with PPFE.

In a previous study, acute exacerbations that occurred after surgical lung biopsy were characterized; the extent of ground-glass opacity in the lung contralateral to the lung from which biopsy samples were acquired was predominantly severe (17). The high-resolution CT findings at the diagnosis of the acute exacerbation in the present case were consistent with a previous report.

Fibroelastosis is a unique pathological feature that involves a combination of both elastosis and collapsed airspace filled with collagen deposition (2). The bronchiolar lesions that were confirmed in the present case included proliferative bronchiolitis, constrictive bronchiolitis obliterans, and peribronchiolar epithelial metaplasia. Table 2 summarizes the previously reported pathological findings in pa- tients with idiopathic PPFE who underwent surgical lung biopsy or autopsy $(1,3,4,6,14,18-36)$. However, the clinicopathological correlation of these bronchiolar lesions with idiopathic PPFE has not been rigorously investigated. Bronchiolar lesions in idiopathic PPFE were clearly described as "identifiable" in 3 studies $(1,18,32)$, and as "unidentifiable" in another (14). In most reports, the presence or absence of bronchiolar lesions was not described. Thus, inattention to bronchiolar lesions in this context may result in the underreporting of bronchiolar lesions.

The coexistence of a PPFE pattern and bronchiolitis obliterans has been reported and emphasized in patients who received lung or bone marrow transplants $(8,37)$. Ofek et al. (8) described the relationship between the PPFE pattern and bronchiolitis obliterans in lung transplant recipients as restrictive allograft syndrome. That is, a PPFE pattern and bronchiolar lesions may share some remodeling processes as a result of lung injuries due to immunologic rejection in association with chronic allograft dysfunction. 


$\begin{array}{llll}\mathrm{VC} & 2.41 & \mathrm{~L} & \\ \% \mathrm{VC} & 65 & \% \\ \mathrm{FVC} & 2.41 & \mathrm{~L} \\ \% \mathrm{FVC} & 67 & \% \\ \mathrm{FEV}_{1} & 1.98 & \mathrm{~L} \\ \% \mathrm{FEV}_{1} & 67 & \% \\ \mathrm{FEV}_{1} / \mathrm{FVC} & 82.2 & \% \\ \dot{\mathrm{V}}_{50} & 3.06 & \mathrm{~L} / \mathrm{s} \\ \dot{\mathrm{V}}_{50} & 81 & \% \\ \dot{\mathrm{V}}_{25} & 0.79 & \mathrm{~L} / \mathrm{s} \\ \% \dot{\mathrm{V}}_{25} & 56 & \% \\ \dot{\mathrm{V}}_{50} / \dot{\mathrm{V}}_{25} & 3.87 & \\ \mathrm{MMF} & 1.96 & \mathrm{~L} / \mathrm{s} \\ \% \mathrm{MMF} & 68 & \% \\ \mathrm{TLC} & 3.40 & \mathrm{~L} \\ \% \mathrm{TLC} & 64 & \% \\ \mathrm{RV} & 0.99 & \mathrm{~L} \\ \mathrm{RV} / \mathrm{TLC} & 29 & \% \\ \% R V / T L C & 94 & \% \\ \% \mathrm{DLCO} & 76 & \%\end{array}$

Figure 3. Pulmonary function testing showed restrictive ventilatory defects with impaired gas exchange. DLco: diffusing capacity of the lung carbon monoxide, $\mathrm{FEV}_{1}$ : forced expiratory volume in one second, FVC: forced vital capacity, MMF: maximal mid-expiratory flow, RV: residual volume, TLC: total lung capacity, VC: vital capacity

It is possible that the bronchiolar lesions in the biopsy specimens in the present case reflect the PFT results, as a component of an obstructive ventilatory defect is partially detected on pathophysiological examination. The concave flow-volume loop during the expiration phase of $25 \%$ to $75 \%$ of FVC might pathophysiologically suggest the existence of small airway abnormalities, although a restrictive ventilatory defect due to interstitial fibrosis definitively diagnosed based on the categorical definition of PFT. In addition, because the present patient was a very light smoker, and because no emphysema was identified on high resolution CT, the impact of smoking on the PFT results was considered to be trivial.

Some studies have reported that a reduced residual volume / total lung capacity ratio (RV/TLC) is a characteristic feature of PFT in PPFE $(7,15)$; however, it is not mandatory for the diagnosis (14). It remains unknown whether PPFE patients, especially those with PPFE accompanied by bronchiolar lesions, exhibit a similar feature because the of value of the RV/TLC ratio has been poorly discussed in previous reports $(1,8,18,32)$.

In general, the pathological features of bronchiolar lesions imply that epithelial injuries occur not only via adverse immune responses in the bronchovascular bundle, including collagen vascular disease and transplantation, but also via inhalation, including hypersensitivity pneumonitis, dust exposure, and some infectious diseases such as mycobacteriosis and fungal conditions. It has recently been suggested that idiopathic PPFE consists of heterogeneous etiologies, and

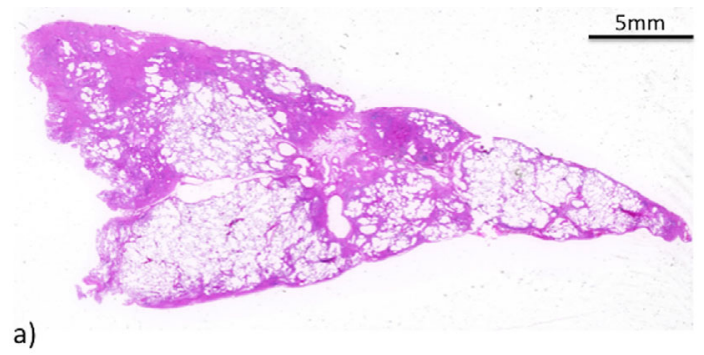

b)

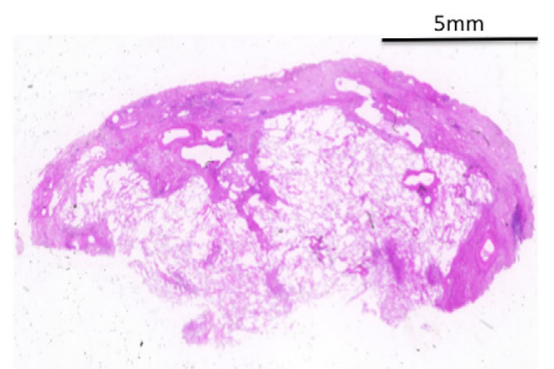

Figure 4. Surgical lung biopsy specimens (Hematoxylin and Eosin staining). (a) Left $\mathbf{S}^{9}$. Subpleural/perilobular fibrosis with abrupt change from normal lung to fibrotic lesions, consistent with a usual interstitial pneumonia pattern. (b) Left $\mathbf{S}^{1+2}$. Subpleural dense, band-like fibrosis, consistent with fibroelastosis. Some fibrotic lesions with bronchiolocentric distribution, separated from the subpleural fibrotic lesions.

are often associated with one or more clinical inhalation factors, such as asbestos, smoking, and organic dust (e.g., mold, bird feces) (1). Drug-related, and autoimmune mechanism-related associations have been hypothesized, as have associations with gastroesophageal reflux and predisposing genetic mechanisms (1). However, in many cases, a diagnosis of idiopathic PPFE is made because it is difficult to prove clinical causation based on the available evidence $(1,3)$. The present case was idiopathic PPFE due to a lack of any clinically relevant cause; however, there are two possibilities that are worth considering. First, the patient was an electrician. The patient declared that he only rarely worked outdoors, and denied occupational exposure to potentially hazardous agents. However, occult inhalation could not be completely excluded. Second, the slight elevation of the serum rheumatoid factor levels may explain the various bronchiolar lesions that were observed in this case, because rheumatoid arthritis has a clear association with bronchiolar lesions and may become clinically overt in the future, although no pathologic findings suggestive of rheumatoid arthritis-including abundant lymphoid aggregation or inflammatory cell interstitial infiltration-were observed in the biopsy specimen $(1,4,38,39)$.

The bronchiolar lesions in this case did not enable a confident clinical diagnosis of idiopathic PPFE or the conclusive determination of any etiologies of a PPFE pattern. However, pathologically proven bronchiolar lesions may be associated with the pathophysiological development or progression to idiopathic PPFE and may indicate that the condi- 

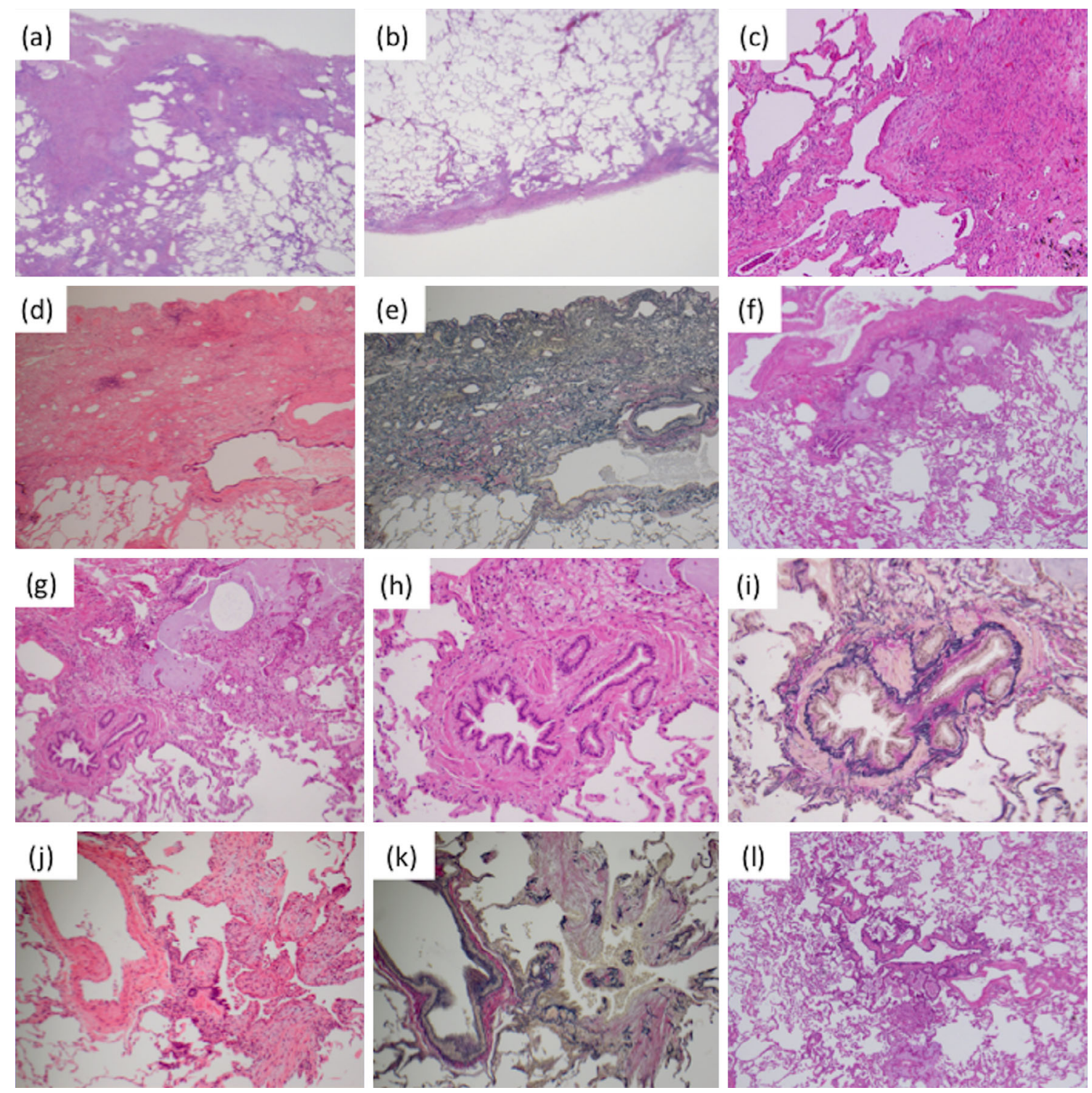

Figure 5. Microscopic findings. (a) Hematoxylin and Eosin $(H \& E)$ staining $\times 2$. Abrupt change from the normal lung to the fibrotic lesion. Perilobular fibrosis and dense fibrosis. (b) H\&E staining $\times 2$. Subpleural fibrosis. (c) H\&E staining $\times 10$. Fibroblastic foci. (d) H\&E staining $\times 4$. (e) Elastica van Gieson (EVG) staining $\times 4$. Fibroelastosis. (f) H\&E staining $\times 2$. (g) H\&E staining $\times 4$. (h) H\&E staining $\times 10$. (i) EVG staining $\times 10$. Constrictive bronchiolitis obliterans with exudate and foamy macrophages was observed infiltrating into the alveolar spaces in the peripheral lobule. (j) H\&E staining $\times 10$. (k) EVG staining $\times 10$. Proliferative bronchiolitis, that is, intraductal polypoid fibrosis that was mainly distributed to the bronchiolar lumen, with quite limited extension to the adjacent peribronchiolar alveolar airspaces. (l) $\mathrm{H} \& \mathrm{E}$ staining $\times 4$. Peribronchial metaplasia.

tion is derived from alternative causes, such as occupational exposure, hypersensitivity pneumonitis, pneumoconiosis, drug-induced lung injury, or collagen vascular disease (e.g., rheumatoid arthritis). In such situations, it is advisable that physicians review the clinical data again in an effort to identify any of the aforementioned alternative causes.

\section{Conclusion}

In summary, in the current patient with idiopathic PPFE, bronchiolar lesions combined with a PPFE pattern might have been associated with the development of idiopathic
PPFE, and might not have been an incidental finding. Bronchiolar lesions may have a close association with PPFE and might help identify any relevant clinical causation.

This case study was approved by the local ethics committee of Toranomon Hospital.

The authors state that they have no Conflict of Interest (COI).

\section{Acknowledgement}

The authors wish to express their appreciation to Dr. Eiyasu Tsuboi (Jizankai Medical Foundation, Tsuboi Cancer Center 
Table 2. Summary of Literature Reports.

\begin{tabular}{|c|c|c|c|c|c|c|c|c|}
\hline \multirow[b]{2}{*}{ Reference } & \multirow[b]{2}{*}{$\mathrm{n}$} & \multirow{2}{*}{$\begin{array}{c}\text { Age } \\
\text { (years) }\end{array}$} & \multirow{2}{*}{$\begin{array}{l}\operatorname{Sex} \\
(\mathrm{m} / \mathrm{f})\end{array}$} & \multicolumn{5}{|c|}{ Pathological findings } \\
\hline & & & & $\begin{array}{c}\text { Subpleural } \\
\text { fibroelasatosis }\end{array}$ & $\begin{array}{l}\text { Bronchocentric } \\
\text { fibrosis }\end{array}$ & $\begin{array}{l}\text { Bronchiolo- } \\
\text { ectasis }\end{array}$ & $\begin{array}{l}\text { Bronchiolitis } \\
\text { obliterans }\end{array}$ & $\begin{array}{c}\text { Fibrosis pattern in } \\
\text { lower lobe }\end{array}$ \\
\hline (4) & 3 & $30-44$ & $2 / 1$ & Identifiable (3/3) & ND & ND & ND & ND \\
\hline (18) & 2 & $43 / 39$ & $0 / 2$ & Identifiable $(2 / 2)$ & ND & Identifiable $(2 / 2)$ & ND & ND \\
\hline (19) & 1 & 27 & $1 / 0$ & Identifiable & ND & ND & ND & $\mathrm{UIP} / \mathrm{p}$ \\
\hline (20) & 7 & $25-83$ & $5 / 2$ & Identifiable & ND & ND & ND & $\mathrm{UIP} / \mathrm{p}(\mathrm{n}=2)$ \\
\hline (36) & 1 & 74 & $1 / 0$ & Identifiable & ND & ND & ND & $\mathrm{UIP} / \mathrm{p}$ \\
\hline (21) & 1 & 85 & $0 / 1$ & Identifiable & ND & ND & ND & Normal lung \\
\hline (22) & 1 & 55 & $1 / 0$ & Identifiable & ND & ND & ND & $\mathrm{NSIP} / \mathrm{p}$ \\
\hline (23) & 2 & $32 / 65$ & $0 / 2$ & Identifiable & ND & ND & ND & ND \\
\hline (24) & 2 & $51 / 59$ & $0 / 2$ & Identifiable & ND & ND & ND & ND \\
\hline (6) & 1 & 82 & $1 / 0$ & Identifiable & ND & ND & ND & ND \\
\hline (25) & 1 & $50 \mathrm{~s}$ & $0 / 1$ & Identifiable & ND & ND & ND & ND \\
\hline (26) & 1 & 49 & $1 / 0$ & Identifiable & ND & ND & ND & Normal lung \\
\hline (1) & $11^{\#}$ & $33-85$ & $5 / 6$ & Identifiable (11/11) & Identifiable (10/11) & ND & ND & Described $\$$ \\
\hline (27) & 2 & $68 / 28$ & $2 / 0$ & Identifiable & ND & ND & ND & ND \\
\hline (29) & 9 & $43-81$ & $4 / 5$ & Identifiable (9/9) & ND & ND & ND & ND \\
\hline (30) & 1 & $60 \mathrm{~s}$ & $0 / 1$ & Identifiable & ND & ND & ND & $\mathrm{PPFE} / \mathrm{p}$ \\
\hline (28) & 1 & 76 & $0 / 1$ & Identifiable & ND & ND & ND & UIP \\
\hline (31) & 1 & 50 & $0 / 1$ & Identifiable & ND & ND & ND & ND \\
\hline (14) & 9 & $19-70$ & $6 / 3$ & Identifiable & Unidentifiable & Unidentifiable & Unidentifiable & ND \\
\hline (32) & 1 & 59 & $0 / 1$ & Identifiable & Unidentifiable & Identifiable & Identifiable & PPFE/p \\
\hline (3) & 5 & $58-87$ & $1 / 4$ & Identifiable & ND & ND & ND & $\mathrm{UIP} / \mathrm{p}(\mathrm{n}=1)$ \\
\hline (33) & 1 & 68 & $1 / 0$ & Identifiable & ND & ND & ND & $\mathrm{UIP} / \mathrm{p}$ \\
\hline (34) & 1 & 41 & $0 / 1$ & Identifiable & ND & ND & ND & ND \\
\hline (35) & $1^{\#}$ & 67 & $1 / 0$ & Identifiable & ND & ND & ND & $\mathrm{UC}(\mathrm{n}=1)$ \\
\hline Current case & 1 & 63 & $1 / 0$ & Identifiable & Identifiable & Identifiable & Identifiable & $\mathrm{UIP} / \mathrm{p}$ \\
\hline
\end{tabular}

\#One case was excluded from the original set of cases due to a definite diagnosis of hypersensitivity pneumonitis.

${ }^{\&}$ Intra-alveolar fibrosis and elastosis $(n=3), U I P / p(n=3)$.

ND: not described, UIP/p: usual interstitial pneumonia pattern, PPFE/p: pleuroparenchymal fibroelastosis pattern, UC: unclassified

Hospital), who provided great assistance in the interpretation of the PFT results.

\section{References}

1. Reddy TL, Tominaga M, Hansell DM, et al. Pleuroparenchymal fibroelastosis: a spectrum of histopathological and imaging phenotypes. Eur Respir J 40: 377-385, 2012.

2. Cheng SK, Chuah KL. Pleuroparenchymal Fibroelastosis of the Lung: a review. Arch Pathol Lab Med 140: 849-853, 2016.

3. Rosenbaum JN, Butt YM, Johnson KA, et al. Pleuroparenchymal fibroelastosis: a pattern of chronic lung injury. Hum Pathol 46: 137-146, 2015.

4. Amitani R, Niimi A, Kuse F. Idiopathic pulmonary upper lobe fibrosis (IPUF). Kokyu 11: 693-699, 1992 (in Japanese).

5. Oda T, Ogura T, Kitamura H, et al. Distinct characteristics of pleuroparenchymal fibroelastosis with usual interstitial pneumonia compared with idiopathic pulmonary fibrosis. Chest 146: 12481255, 2014.

6. Nei T, Kawamoto M, Satoh E, et al. A case of suspected idiopathic pulmonary upper lobe fibrosis (Amitani disease) with acute exacerbation. Nihon Kokyuki Gakkai Zasshi (J Jpn Respir Soc) 47: 116-121, 2009 (in Japanese, Abstract in English).

7. Enomoto Y, Nakamura Y, Satake Y, et al. Clinical diagnosis of idiopathic pleuroparenchymal fibroelastosis: a retrospective multicenter study. Respir Med 133: 1-5, 2017.

8. Ofek E, Sato M, Saito T, et al. Restrictive allograft syndrome post lung transplantation is characterized by pleuroparenchymal fibroe- lastosis. Mod Pathol 26: 350-356, 2013.

9. Harada T, Yoshida Y, Kitasato Y, et al. The thoracic cage becomes flattened in the progression of pleuroparenchymal fibroelastosis. Eur Respir Rev 23: 263-266, 2014.

10. Collard HR, Moore BB, Flaherty KR, et al. Acute exacerbations of idiopathic pulmonary fibrosis. Am J Respir Crit Care Med 176: 636-643, 2007.

11. In: Clinical diagnostic and treatment guidance for idiopathic interstitial pneumonias. 2nd ed. Japanese Respiratory Society's Committee formulating diagnosis and treatment guideline for diffuse lung diseases. Nankodo, Tokyo, 2010 (in Japanese).

12. Epler GR, Colby TV. The spectrum of bronchiolitis obliterans. Chest 83: 161-162, 1983.

13. Churg A, Muller NL, Silva CI, Wright JL. Acute exacerbation (acute lung injury of unknown cause) in UIP and other forms of fibrotic interstitial pneumonias. Am J Surg Pathol 31: 277-284, 2007.

14. Watanabe S, Waseda Y, Takato H, et al. Pleuroparenchymal fibroelastosis: Distinct pulmonary physiological features in nine patients. Respir Investig 53: 149-155, 2015.

15. Ishii H, Watanabe K, Kushima H, et al. Pleuroparenchymal fibroelastosis diagnosed by multidisciplinary discussion in Japan. Respir Med 141: 190-197, 2018.

16. Sato $\mathrm{H}$, Teramukai $\mathrm{S}$, Kondo $\mathrm{H}$, et al. Impact and predictors of acute exacerbation of interstitial lung diseases after pulmonary resection for lung cancer. J Thorac Cardiovasc Surg 147: 1604-1611, 2014.

17. Kondoh Y, Taniguchi H, Kitaichi M, et al. Acute exacerbation of 
interstitial pneumonia following surgical lung biopsy. Respir Med 100: 1753-1759, 2006.

18. Jingu K, Kawana A, Furihata K, et al. Two cases of marked pulmonary fibrosis in the upper lung field. Kokyu 18: 318-323, 1999 (in Japanese).

19. Kobayashi Y, Sakurai M, Kushiya M, et al. Idiopathic pulmonary fibrosis of the upper lobe: a case report. Nihon Kokyuki Gakkai Zasshi (J Jpn Respir Soc) 37: 812-816, 1999 (in Japanese, Abstract in English).

20. Shiota S, Shimizu K, Suzuki M, et al. Seven cases of marked pulmonary fibrosis in the upper lobe. Nihon Kokyuki Gakkai Zasshi (J Jpn Respir Soc) 37: 87-96, 1999 (in Japanese, Abstract in English).

21. Kobashi Y, Ohba H, Yoneyama H, Okimoto N, Sakamoto K, Soejima R. A case of so-called "idiopathic pulmonary upper lobe fibrosis" complicated by both mediastinal emphysema and bilateral pneumothorax at different times. Kokyu 19: 292-298, 2000 (in Japanese).

22. Kamoi H, Okamoto T, Yoshimura N, et al. [A case of interstitial pneumonia in the upper lung field histologically diagnosed as nonspecific interstitial pneumonia complicated by bilateral pneumothorax]. Nihon Kokyuki Gakkai Zasshi (J Jpn Respir Soc) 40: 936940, 2002 (in Japanese, Abstract in English).

23. Frankel SK, Cool CD, Lynch DA, Brown KK. Idiopathic pleuroparenchymal fibroelastosis: description of a novel clinicopathologic entity. Chest 126: 2007-2013, 2004.

24. Becker CD, Gil J, Padilla ML. Idiopathic pleuroparenchymal fibroelastosis: an unrecognized or misdiagnosed entity? Mod Pathol 21: 784-787, 2008.

25. Satoh S, Kawai S, Shirai T, Isogai S, Taura S, Nakaminato S. A case of idiopathic pulmonary upper lobe fibrosis. Rinsho Hoshasen 55: 211-214, 2010 (in Japanese).

26. Morimoto A, Mochizuki Y, Nakahara Y, Kawamura T, Sasaki S, Kobashi Y. A case of idiopathic pulmonary upper lobe fibrosis. Nihon Kokyuki Gakkai Zasshi (J Jpn Respir Soc) 48: 944-949, 2010 (in Japanese, Abstract in English).

27. Piciucchi S, Tomassetti S, Casoni G, et al. High resolution CT and histological findings in idiopathic pleuroparenchymal fibroelastosis: features and differential diagnosis. Respir Res 12: 111-115, 2011.

28. Noh HJ, Seo Y, Huo SM, Kim TJ, Kim HL, Song JS. Idiopathic pleuroparenchymal fibroelastosis presenting in recurrent pneumot- horax: a case report. Tuberc Respir Dis (Seoul) 77: 184-187, 2014. 29. Watanabe K, Nagata N, Kitasato Y, et al. Rapid decrease in forced vital capacity in patients with idiopathic pulmonary upper lobe fibrosis. Respir Investig 50: 88-97, 2012.

30. Kudoh K, Takahashi R, Shimoyama N. An autopsy case of idiopathic pulmonary upper lobe fibrosis (Amitani disease). Shindan Byori 31: 213-217, 2014 (in Japanese).

31. Cuppens K, Verbeken E, Coolen J, Verschakelen J, Wuyts W. Idiopathic pleuroparenchymatous fibroelastosis: a case report and brief review of the literature. Respir Med Case Rep 127-129, 2014.

32. English JC, Mayo JR, Levy R, Yee J, Leslie KO. Pleuroparenchymal fibroelastosis: a rare interstitial lung disease. Respirol Case Rep 3: 82-84, 2015.

33. Sato S, Hanibuchi M, Takahashi $M$, et al. A patient with idiopathic pleuroparenchymal fibroelastosis showing a sustained pulmonary function due to treatment with pirfenidone. Intern Med 55: 497-501, 2016.

34. Thangakunam B, Isaac BT, Christopher DJ, Burad D. Idiopathic pleuroparenchymal fibroelastosis - a rare idiopathic interstitial pneumonia. Respir Med Case Rep 17: 8-11, 2016.

35. Gomes PS, Shiang C, Szarf G, Coletta EN, Pereira CA. Pleuroparenchymal fibroelastosis: report of two cases in Brazil. J Bras Pneumol 43: 72-75, 2017.

36. Sakamoto A, Kuwano K, Komori M, et al. A case with usual interstitial pneumonia characterized by subpleural fibrosis predominantly in the bilateral upper lobe. Nihon Kyobu Rinsho (Jpn J Chest Dis) 59: 628-631, 2000 (in Japanese).

37. von der Thusen JH, Hansell DM, Tominaga M, et al. Pleuroparenchymal fibroelastosis in patients with pulmonary disease secondary to bone marrow transplantation. Mod Pathol 24: 1633-1639, 2011.

38. Portillo K, Arriaga IG, Ruiz-Manzano J. Pleuroparenchymal fibroelastosis: is it also idiopathic entity? Arch Broncopneumol 51: 509-514, 2015.

39. Lin E, Limper AH, Mona T. Obliterative bronchiolitis associated with rheumatoid arthritis: analysis of a single center case series. BMC Pulm Med 18: 105, 2018.

The Internal Medicine is an Open Access journal distributed under the Creative Commons Attribution-NonCommercial-NoDerivatives 4.0 International License. To view the details of this license, please visit (https://creativecommons.org/licenses/ by-nc-nd/4.0/).

(C) 2019 The Japanese Society of Internal Medicine

Intern Med 58: 1321-1328, 2019 